\title{
Investigation of a Robust Remote Heterodyne Envelope Detector Scheme for Cost-Efficient E-PON/60GHz Wireless Integration
}

\author{
Spiros Mikroulis, Manoj P Thakur, John E Mitchell \\ University College London (UCL), Electronic \& Electrical Engineering, Torrington Place, WC1E 7JE, London, \\ United Kingdom
}

\begin{abstract}
In this work, a simple remote heterodyne envelope detector scheme is proposed employing an O-SSB scheme for application in a converged E-PON/60GHz scenario. The proposed PON-60GHz wireless system topology (Fig. 1) is discussed and an evaluation of this scheme is experimentally demonstrated including i) an OFDM $60 \mathrm{GHz}$ wireless access system using indoor MMF, ordinary DFB lasers \& ii) a $60 \mathrm{GHz}$ wireless PON "bridge" using a VCSEL for the E-PON data. In both cases, the envelope detector scheme proves its robustness against phase/ wavelength variations while it preserves low cost and wavelength tolerance.
\end{abstract}

Keywords: radio over fiber, mm wave communications, passive optical network

\section{INTRODUCTION}

In the last few years, the license-free spectrum at $60 \mathrm{GHz}$, which offers (in accordance to FCC) an available bandwidth of $7 \mathrm{GHz}$ has gained much attention so as to satisfy the emerging bandwidth-hungry front/backhauling or access applications. In this band, Radio over Fiber (RoF) technology [1] offers simplification of base station (BS) sites through the introduction of remote antenna units (RAUs) connected to a central base station (CBS),

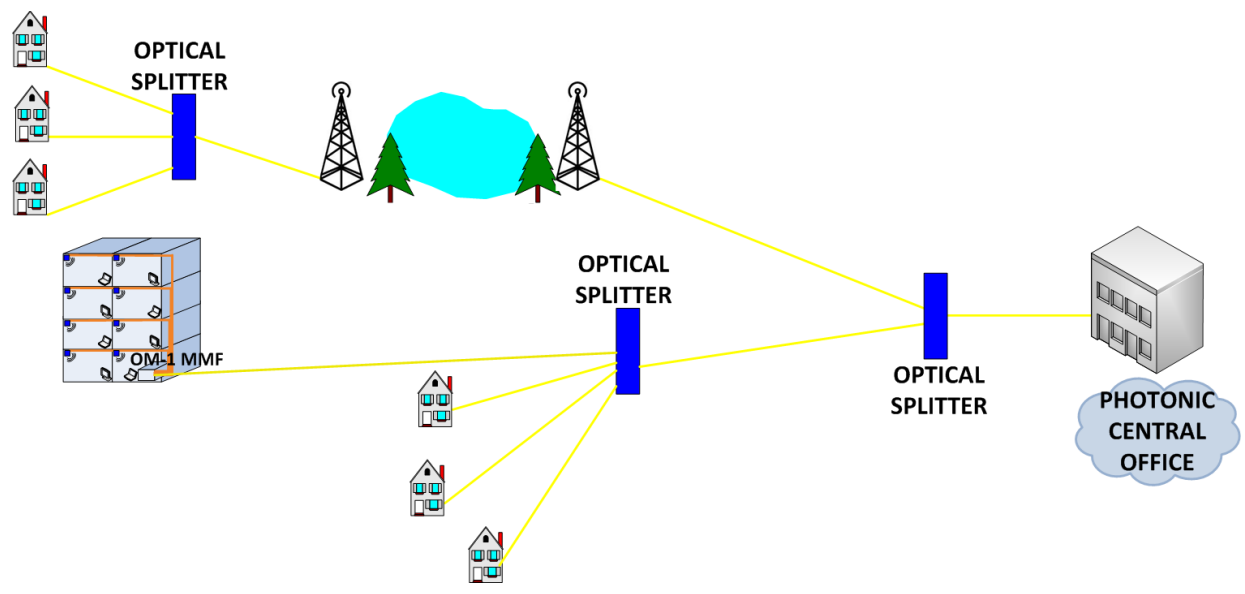

Figure 1. Converged PON-wireless system topology addressing 60GHz radio over fiber, standard E-PON, and $60 \mathrm{GHz}$ wireless bridge supporting E-PON virtual extension.

using already deployed PON-FTTx. In this case, the exploitation of the RoF technology at a high density indoor $60 \mathrm{GHz}$ distributed antenna system, eliminates the needs for local oscillators (LOs), digital signal processors (DSPs), and mixers at the RAUs, potentially leading to a few tens of Watts lower power per RAU, while on the other hand it significantly reduces the overall cost in terms of CapEx/OpEx. In areas where fiber infrastructure is not available, $60 \mathrm{GHz}$ can be used for PON front/backhauling, serving as wireless virtual bridge between optical links. Figure 1 shows a proposed converged fiber-wireless network architecture, supporting 60GHz radio over fiber indoor wireless access, standard E-PON, and a $60 \mathrm{GHz}$ wireless bridge providing a virtual E-PON extension. The major factors which will determine the real deployment of this scenario are; the simplicity and compatibility of the components used with the standard PON devices, the robustness against wavelength variations (i.e. colorless transceiver design) and the overall cost in terms of CapEx/OpEx.

In this work, a simple remote heterodyne scheme utilizing two optical tones using ordinary long wavelength lasers is proposed for $\mathrm{mm}$ wave generation as to provide significant advantages in terms of simplicity, compatibility with standardized long wavelength PON devices, and low cost due to the absence of high frequency modulators. It is well-known that the phase noise of the carrier generated by the beating of two uncorrelated optical sources may manifest itself in the broadening of the spectral linewidth. Also, in the presence 
of phase noise, a carrier-recovery circuit might have difficulties in tracking rapid phase variations, resulting in degradation of the detection performance. In this paper, a V-band (50-75 GHz) envelope detector is used so as to avoid phase related impairments, while on the other hand if the data carrying wavelength is coming from a thermally uncooled source, the proposed setup can cope with wavelength variations as long as the generated carrier frequency is within the frequency range of the envelope detector (in our case V-band 50-75 GHz). This margin of operation further helps to remove the need to modify or control the PON source, thereby making the system independent and generically compatible with source technology. An evaluation of the proposed scheme is experimentally demonstrated under i) a cost-efficient Gigabit wireless access case using already installed indoor MMF fed by a passive optical network (PON) topology using a conventional (central) SMF-OM-1 MMF launching technique at $1550 \mathrm{~nm}$, and ii) a $60 \mathrm{GHz}$ wireless PON network "bridge" using a uncooled VCSEL for the E-PON data.

\section{DEMONSTRATION OF A SMF-MMF 60GHz RADIO OVER FIBER SYSTEM USING REMOTE HETERODYNE ENVELOPE DETECTOR TECHNIQUE}

Graded index multimode fiber (GI-MMF) has been widely used in cost-sensitive short-reach/indoor applications such as interconnects in datacenters, local area networks (LANs), and cloud computing, where most of the

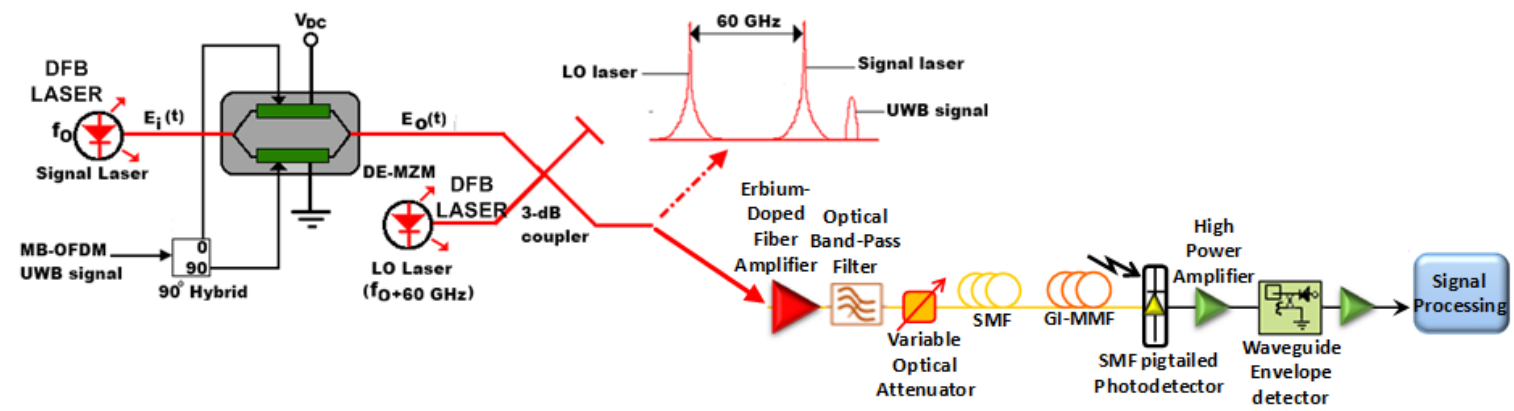

Figure 2. Uncorrelated optical heterodyne scheme used in the SMF-MMF link experiments.

installed building networks are based on OM-n (n=1,2,3,4) MMF topology (90\%) for speeds (up to 10Gb/s), short range (up to $300 \mathrm{~m}$ ) optical networks [2]. MMFs can be easily installed and handled, on the roadmap to the "Do It Yourself" concept. However, the stimulation of a large number of propagation modes in MMFs restricts their maximum available bandwidth-length $(\mathrm{BWxL})$ product due to the modal dispersion effect. Recently, OM-1 GI-MMF has been proposed by the authors as a cost-efficient solution for indoor mm-wave remote antenna deployment in order to re-use the existing MMF infrastructure for 60GHz MB-OFDM signal transmission [3]. In this case, a conventional (central) SMF-OM-1 MMF launching technique at $1550 \mathrm{~nm}$ is applied as to reach a restricted (underfilled) mode launch condition, greatly improving the bandwidth length product. Additionally, a $\mathrm{MMF} / \mathrm{SMF}$ coupling stage is used at the receiver where ordinary components used at $60 \mathrm{GHz}$ (i.e. $70 \mathrm{GHz}$ photodiodes $)$ are typically SMF pigtailed. A two optical tone scheme using ordinary $(\Delta \lambda=10 \mathrm{MHz})$ thermally tuned long wavelength DFB lasers is used for a remote heterodyne detection at a high speed photodiode. The experimental set up is as below (Fig.2);

A UWB (MB-OFDM) signal is generated offline in MATLAB and then loaded into an AWG operating at a sampling rate of $10.8 \mathrm{GS} / \mathrm{s}$. The outputs of the AWG (i.e. the UWB signal and its Hilbert Transform) are amplified by two 16-dB gain, RF amplifiers and then filtered by two 7.5-GHz, LPFs. These filtered signals are then used as the electrical drives to a Fujitsu 10-Gbit/s DE-MZM, biased at its quadrature point (Fig.2). In this case, the MZM is configured as an O-SSB I-Q modulator. A composite scheme consisting of $17.5 \mathrm{~km}$ of SMF and $250 \mathrm{~m}$ of OM-1 MMF was undertaken, so as to demonstrate the application of this technique on a future urban SMF-PON/indoor MMF wired-wireless scenario. A VOA is used to maintain a maximum of $1 \mathrm{dBm}$ input to the $70-\mathrm{GHz}$ broadband photodetector located at the base station. After photodetection, the $60-\mathrm{GHz} \mathrm{UWB}$ signal is amplified by a 30-dB gain, low-noise amplifier (LNA). Down-conversion of the mm-wave UWB signal is achieved by linearly detecting its envelope using a waveguide broadband detector. The down-converted signal is further amplified by a $30-\mathrm{dB}$ gain amplifier with a frequency range of $0.7-4.2 \mathrm{GHz}$ and then captured by a real-time oscilloscope operated at a sampling rate of $50 \mathrm{GS} / \mathrm{s}$. 
The optical system performance is tested for various received optical powers at a raw bit rate of $3.84 \mathrm{~Gb} / \mathrm{s}$. The peak-to-peak amplitudes of the UWB signals from the AWG are kept fixed at $0.6 \mathrm{~V}$. The resulting optical
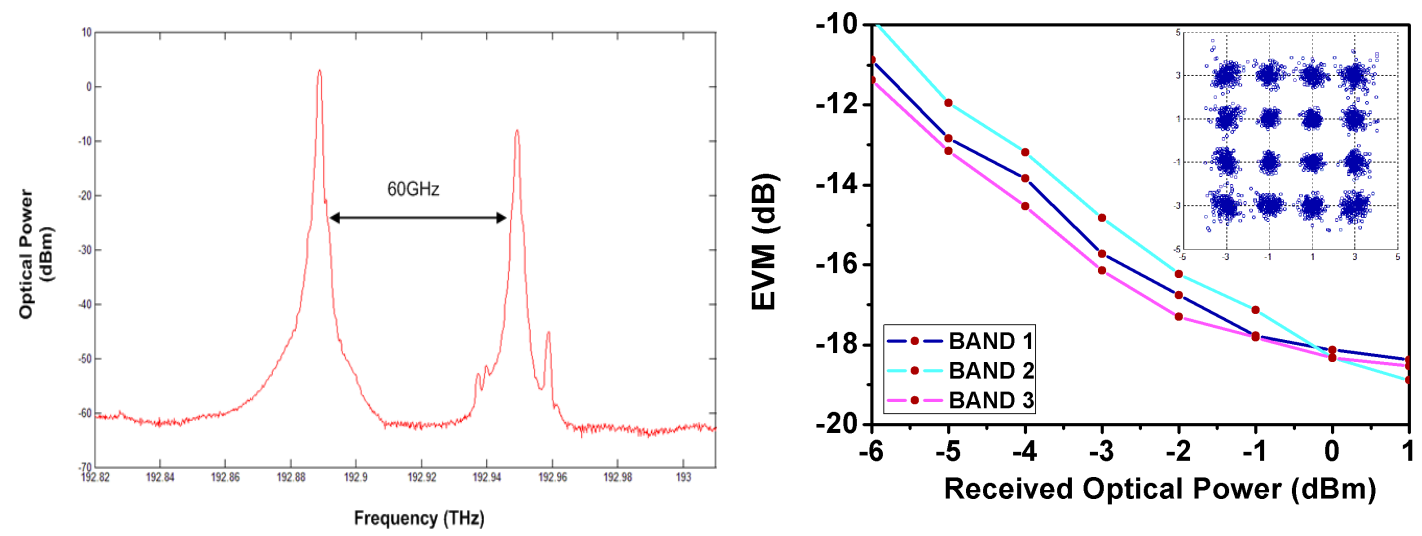

Figure 3. Optical spectrum showing the O-SSB uncorrelated optical heterodyne scheme used in the experiments (left diagram), and measured EVM values of the SMF-MMF link at a bit rate of 3.84Gb/s (right diagram).

spectrum, including the measured EVMs can be observed in Fig 3. The EVM is limited by electrical noise at low received optical power, for all cases. Increasing the received optical power improves the EVM due to an increase in the received signal-to-noise ratio (SNR). However, EVM tends to saturate at high power levels limited by the electrical amplifier transfer function's nonlinearity. An acceptable forward error correction (FEC) aided performance of $-16 \mathrm{~dB}\left(\mathrm{BER}=10^{-3}\right)$ is demonstrated for the link at a received power in the order of $-2 \mathrm{dBm}$ or greater.

\section{DEMONSTRATION OF A REAL TIME BIDIRECTIONAL 60GHz WIRELESS PON "bridge" USING AN UNCOOLED VCSEL FOR THE E-PON DATA AND ENVELOPE DETECTOR TECHNIQUE}

The demonstrated system set up for the wireless PON virtual extension is depicted in Fig. 4. The set up demonstrates a simple and cost-effective $60 \mathrm{GHz}$ wireless scheme to extend and compliment the E-PON capable

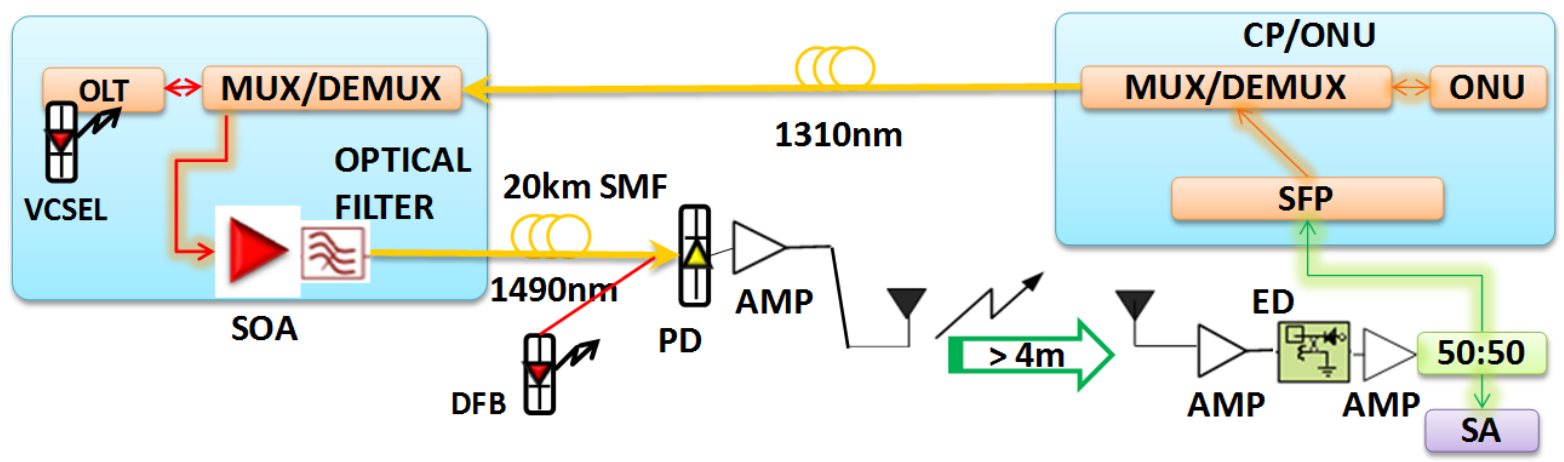

Figure 4. Bidirectional 60GHz wireless PON bridge used in the experiments.

of delivering a data rate up to $1 \mathrm{~Gb} / \mathrm{s}$ per cell in indoor environment. In the implementation a direct detection using a $3 \mathrm{GHz}$ BW V-band waveguide envelope detector is utilized, followed by optical conversion using SFP to provide a transparent wireless link. The setup is tested for both TCP/IP and UDP data packets for any link drops due to arbitrary shifts in the uncooled laser's wavelength, and wireless path interruptions. The resulting optical spectrum, the network's performance and net bandwidth achieved for a duration of 2 hours are depicted in Figure 5. Figure 5 shows that the average sustained data rate for TCP/IP connection was $700 \mathrm{Mb} / \mathrm{s}$ with peaks of $800-900 \mathrm{Mb} / \mathrm{s}$. Ongoing for the UDP connection sustained data rates of $950 \mathrm{Mb} / \mathrm{s}$ are observed for the same duration. Intentional link drops are introduced by blocking the wireless path which can be observed in Fig 5 . The time for the connection re-establishement once the wireless path is clear was in the order of $60 \mathrm{~s}$. 

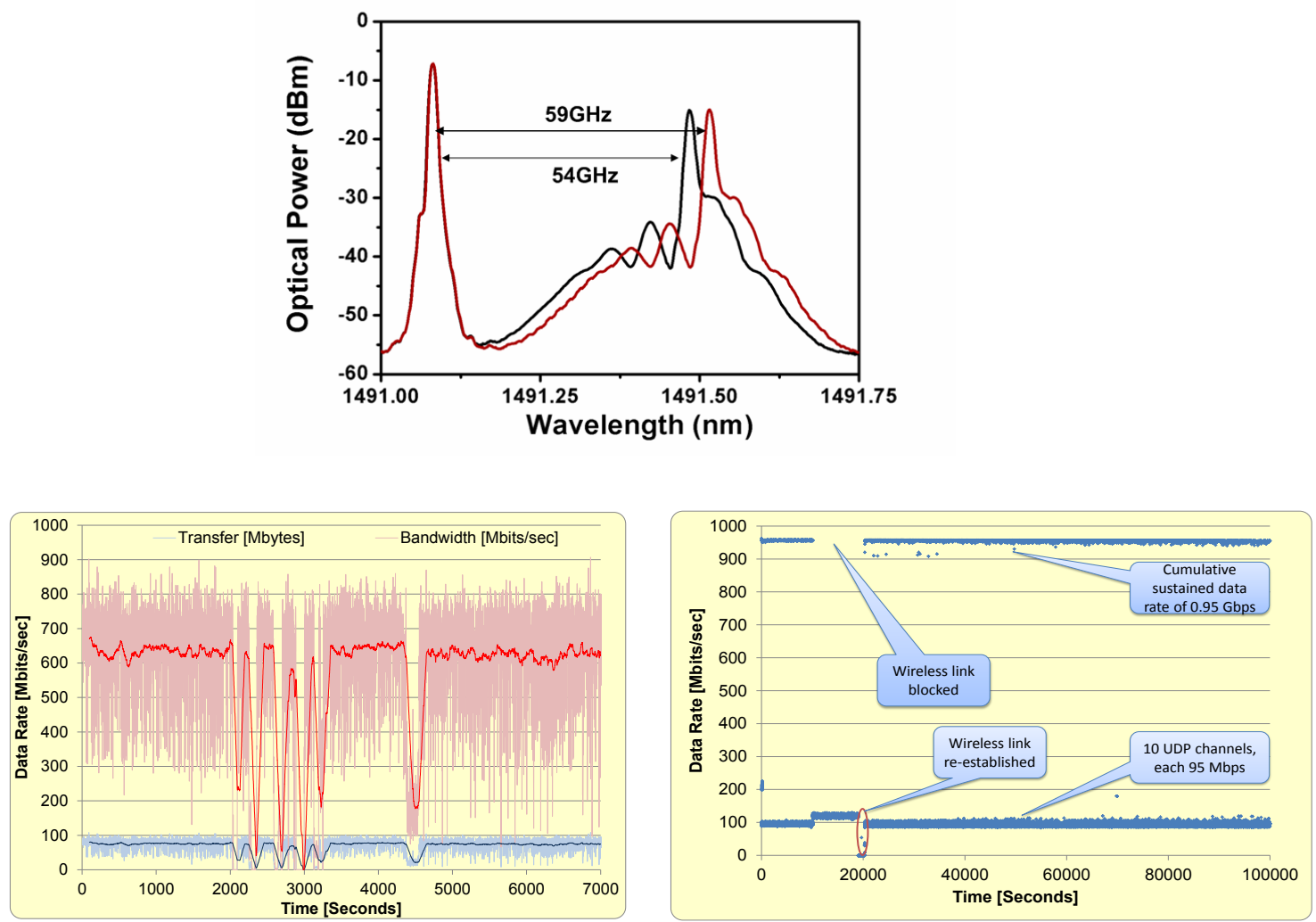

Figure 5. Optical spectrum showing the O-SSB uncorrelated optical heterodyne scheme used in the experiments exhibiting optical tone drifting due to the uncooled VCSEL source (top diagram), TCP/IP data transfer (left diagram), 10 UDP channel data transfer (right diagram).

\section{CONCLUSIONS}

In this paper a robust remote heterodyne envelope detector scheme is proposed for application in a converged E$\mathrm{PON} / 60 \mathrm{GHz}$ scenario. The scheme is experimentally evaluated using a 60GHz SMF-MMF radio over fiber transmission using MB-OFDM, and a $60 \mathrm{GHz}$ wireless PON virtual extension using real data transfer. In both cases the key components of a converged E-PON/60GHz scenario demonstrate its resilience to system imperfections such as wavelength drift, while preserving its simplicity and potential for colorless operation.

\section{ACKNOWLEDGMENT}

The work reported in this paper has been carried out within the Marie Curie Intra European Fellowship (MCIEF) "multimode FIber Radio technology for cost-efficient indoor Mm-WAve REmote antenna systems, (FIRMWARE)" within the 7th European Community Framework Programme (grant agreement No. 327494).

\section{REFERENCES}

[1] M. Parker, S. D. Walker, R. Llorente, M. Morant, M. Beltrán, I. Möllers, D. Jäger, C. Vázquez, D. Montero, I. Librán, S. Mikroulis, S. Karabetsos, A. Bogris, "Radio-over fibre technologies arising from the Building the future Optical Network in Europe (BONE) project”, IET Optoelectronics, vol. 4, No. 6, pp. 247-259, 2010.

[2] A. J. Seeds, and T. Ismail, "Broadband access using wireless over Multimode Fiber Systems", IEEE/OSA J. Light. Technol., vol. 28, No. 16, pp. 2430-2435, 2010.

[3] S. Mikroulis, O. Omomukuyo, M. P. Thakur, and J. E. Mitchell, "Investigation of a SMF-MMF link for a Remote Heterodyne 60-GHz OFDM RoF Based Gigabit Wireless Access Topology”, IEEE/OSA J. Light. Technol., in press, DOI:10.1109/JLT.2014.2322752, 2014. 